\title{
Diversitas Journal
}

ISSN 2525-5215

DOI: $10.17648 /$ diversitas-journal-v3i2.608

Volume 3, Número 2 (mai./ago. 2018 ) pp: 239-244. www.kentron.ifal.edu.br/index.php/diversitas_journal (C) Diversitas Journal

\section{A influência da emoção com o desenvolvimento da doença Vitiligo}

\section{The influence of emotion with the development of Vitiligo disease}

\author{
Sheylla Acacio dos Santos ${ }^{(1)}$; Cleis Nunes Santos ${ }^{(2)}$; Jaqueline Maria da Silva ${ }^{(3)}$
}

Página | 239

\author{
(1)Enfermeira; Faculdade São Vicente de Pão de Açúcar - FASVIPA Email: sheyllaacacio19@gmail.com; \\ (2)Enfermeira; Faculdade São Vicente de Pão de Açúcar - FASVIPA; \\ ${ }^{(3)}$ Pos-doc em Química e Biotecnologia - IQB / UFAL.
}

Todo o conteúdo expresso neste artigo é de inteira responsabilidade dos seus autores.

Recebido em: 30 de maio de 2018; Aceito em: 23 de julho de 2018; publicado em 02 de 09 de 2018. Copyright(C) Autor, 2018.

RESUMO: O vitiligo é uma doença sistêmica crônica, adquirida de evolução clinica imprevisível caracterizado pelo surgimento de maculas e manchas em áreas da pele e mucosas com tendência a aumentar significativamente de tamanho, em decorrência da ausência de melanina por desaparecimento dos melanócitos na área afetada. Sua causa e seu mecanismo bioquímico de ação são desconhecidos. O objetivo principal é descrever à ação do estado emocional no desenvolvimento da doença vitiligo em portadores; facilitando assim a descrição das modificações que os sentimentos podem ocasionar no desenvolvimento desta dermatose. Trata-se de uma revisão descritiva bibliográfica, que busca expor a relação do estado emocional no desenvolvimento da doença vitiligo.A doença afeta diretamente a produção de melanina através dos melanócitosque são células derivados da crista neural. Na maioria dos pacientes com vitiligo, o diagnóstico é feito por exame clínico seguido de análise da área despigmentada sob a luz de Wood. O tratamento tem algumas variantes, pois tem como característica basear-se na complexidade, na extensão da manifestação e depende ainda do estado psicológico e principalmente da cor da pele. Sendo assim, os sentimentos de rejeição, vergonha, frustração e tristeza aliada ao estresse pode gerar um fator muito relevante no desenvolvimento da doença vitiligo. Isso porque está diretamente ligado com o sistema nervoso, que por sua vez, o aumento desses sentimentos eleva na produção de hormônios neurotransmissores e neuroendócrinos, responsáveis respectivamente pela transmissão de estímulos e regulação do organismo.

PALAVRAS-CHAVE: Vitiligo; Psicodermatologia e Epiderme.

\begin{abstract}
Vitiligo is a chronic systemic disease, acquired from an unpredictable clinical evolution characterized by the appearance of maculas and spots in areas of the skin and mucous membranes with a tendency to increase significantly in size, due to the absence of melanin due to disappearance of the melanocytes in the affected area. Its cause and its biochemical mechanism of action are unknown. The main objective is to describe the action of the emotional state in the development of vitiligo disease in patients; thus facilitating the description of the changes that the feelings can cause in the development of this dermatosis. This is a descriptive bibliographical review, which seeks to expose the relationship of the emotional state in the development of vitiligo disease. The disease directly affects the production of melanin through the melanocytes that are cells derived from the neural crest. In most patients with vitiligo, the diagnosis is made by clinical examination followed by analysis of the depigmented area under the light of Wood. The treatment has some variants, since it has as characteristic to be based in the complexity, in the extension of the manifestation and still depends on the psychological state and mainly of the color of the skin. Thus, the feelings of rejection, shame, frustration and sadness allied to stress can generate a very relevant factor in the development of vitiligo disease. This is because it is directly connected with the nervous system, which in turn, the increase of these feelings raises in the production of neurotransmitter and neuroendocrine hormones, responsible respectively for the transmission of stimuli and regulation of the organism.
\end{abstract}

KEYWORDS: Vitiligo; Psicodermatologia e Epiderme. 


\section{INTRODUÇÃO}

A emoção, em um estudo etimológico da palavra evidenciou-se que ela deriva de duas outras palavras do latim "exmovere", que significa em movimento. Então, quando estamos "emocionados" o nosso corpo se movimenta internamente e externamente, o que faz sentido ao seu significado etimológico (CASANOVA et al., 2009).

O vitiligo é uma dermatose que não leva à incapacidade funcional, mas causa grande impacto psicossociocultural. Pode ser desfigurante, influindo negativamente na autoestima da pessoa, sobretudo nos casos extensos e em pessoas de pele negra (SILVA et al., 2009).

A doença pode estar representada em emoções não resolvidas que se apresentam, então, como manifestações orgânicas. "O sintoma vem como manifestação simbólica, como linguagem do que permaneceu oculto, excluído de poder manifestar-se" (AZULAY, 1997 apud DIAS, 2005).

Em uma abordagem sobre o vitiligo evidenciou-se que... "O estado emocional do paciente como angustia, tristeza e raiva podem interferir no tratamento, pois alterações emocionais desequilibram o organismo" (NETO et al., 2015).

A convivência com doenças crônicas como o vitiligo modifica o cotidiano das pessoas fazendo surgir sentimentos como vergonha e culpa que podem trazer consequências sobre a percepção da qualidade de vida por parte dos acometidos e de suas famílias (SILVA et al., 2009).

A pergunta que norteou esse trabalho foi: Existe alguma interação entre as emoções que os portadores da doença vitiligo apresentam com o seu desenvolvimento e tratamento, e de que forma elas podem influenciar?

O estudo proporcionará um aprofundamento acerca dos conhecimentos do desenvolvimento da doença Vitiligo, evidenciando suas características, visando uma melhoria no conhecimento dos profissionais de saúde, tornando a doença mais evidente perante a sociedade e convívio social (SILVA, 2015). Buscando assim uma melhor interação entre portadores e familiares, uma vez que a doença não é contagiosa nem tão pouco infecciosa. Observa-se que, além dos fatores já conhecidos e prováveis para o desenvolvimento da doença Vitiligo, os fatores emocionais podem contribuir nesse processo, havendo então a necessidade de uma pesquisa para a compreensão dessa ação. 


\section{PROCEDIMENTO METODOLÓGICO}

O estudo trata-se de uma revisão descritiva bibliográfica, que busca descrever a interação entre as emoções que os portadores da doença vitiligo apresentam com o seu desenvolvimento e tratamento, e de que forma influencia na sua vida.

Foram realizados levantamentos bibliográficos com autores que abordaram sobre o assunto nas seguintes bases de dados: Scielo, Lilacs, Pubmed, Google acadêmico, Science Direct e Web off Science; 25 artigos foram selecionados por abordarem sobre o vitiligo com olhar voltado a influência do estado emocional dos portadores no desenvolvimento e tratamento da doença, sendo publicados entre os anos de 2012 a 2016.

Critérios de inclusão:- Publicados em português; - Publicados no período entre 2012 a 2016; - Disponíveis na íntegra gratuitos.

Critérios de exclusão: -Publicados em línguas estrangeiras; - Publicados em ano anterior a 2012; -Arquivos que não estão disponíveis na íntegra.

\section{RESULTADOS E DISCUSSÃO}

Ao analisar, separadamente, cada um dos descritores, encontrou-se um elevado número de publicações, os quais estão listados na tabela a seguir.

Tabela 1: Número de publicações na Biblioteca Virtual de Saúde segundo descritores

\begin{tabular}{lllll}
\hline Descritores & Scielo & Pubmed & Lilacs & $\begin{array}{l}\text { Google } \\
\text { acadêmico }\end{array}$ \\
\hline Vitiligo & 59 & 6.060 & 253 & 2.620 \\
Pele & 2.659 & 290 & 9.120 & 191 \\
$\begin{array}{l}\text { Representação Social } \\
\text { Psicodermatologia }\end{array}$ & 1.028 & 01 & 1.088 & 373 \\
& 250 & 370 & 587 & 1.187 \\
Epiderme & 575 & 32 & 387 & 24.800 \\
\hline TOTAL & 4.571 & 6.753 & 11.435 & 29.171 \\
\hline
\end{tabular}

Fontes: Scielo; Pubmed; Lilacs e Google acadêmico. 
Diante dos dados expostos na tabela 1 é nítida a prevalência do Google Acadêmico quando da busca por descritores, totalizando 29.171 publicações.

Em concordância com Silva (2013), realizou-se a associação dos descritores citados a seguir, a fim de realizar uma aproximação das produções encontradas, ou seja, daquelas que contribuíssem para elucidação do objetivo apresentado.

Para obtenção de mais detalhes de publicações que tem uma abordagem que se volta de forma mais explicita ao tema escolhido neste trabalho, elaborou-se uma tabela com descritores associados em dupla.

Tabela 2: Número de publicações na Biblioteca Virtual de Saúde segundo descritores associados em dupla.

\begin{tabular}{|c|c|c|c|c|c|}
\hline Descritores & Scielo & Pubmed & Lilacs & $\begin{array}{l}\text { Google } \\
\text { acadêmico }\end{array}$ & Total \\
\hline Emoções e pele & 01 & 02 & 02 & 03 & 08 \\
\hline $\begin{array}{l}\text { Representação social } \\
\text { e vitiligo }\end{array}$ & 02 & 01 & 02 & 01 & 06 \\
\hline $\begin{array}{l}\text { Epiderme } \\
\text { psicodermatologia }\end{array}$ & 02 & 03 & 01 & 05 & 11 \\
\hline Total geral & 05 & 06 & 05 & 09 & 25 \\
\hline
\end{tabular}

Fontes: Scielo; Pubmed; Lilacs e Google acadêmico.

Do total de 30 produções científicas, 25 artigos foram analisados e pode-se afirmar que $70 \%$ possuem uma abordagem qualitativa; $15 \%$ abordam o assunto de forma quantitativa; $10 \%$ de ordem integrativa e $5 \%$ documental.

O estudo realizado possibilitou de forma explicita identificar que de acordo com o que "se sente", o indivíduo portador da doença Vitiligo pode interferi em seu tratamento e até mesmo influenciar no seu desenvolvimento (ANDRADEet al., 2016). Segundo Dias et al., (2016) demonstram que os fatores desencadeantes em dermatoses crônicas de crianças em especial com Vitiligo são internalizados ou externalizados em função das ações emocionais vividas por esses indivíduos, assim demonstram que mães de crianças com Vitiligo atribuíram como fatores desencadeantes a interação da criança com mãe/pai e fatores emocionais.

A adaptação positiva na doença crônica também está associada à presença de fatores que de acordo com Castro \& Moreno-Jimenez (2007) apud MENEZES et al., (2010) podem ser compreendidos como: (a) Fatores de risco: Variedade da doença(gravidade, visibilidade, problemas médicos associados); nível de funcionamento que não depende da criança; e estressores psicossociais que se relacionam com a doença; (b) Fatores de proteção: variáveis intrapessoais (temperamento, habilidade para resolver problemas); ambiente familiar; apoio social; recursos da comunidade; habilidades no processamento do estresse (estratégias de elaboração de enfrentamento). 
O estresse foi detectado como um fator negativo no desenvolvimento da doença, uma vez que mexe com o psicológico e sistema nervoso do paciente, isso porque os hormônios neuroendócrinos e neurotransmissores autônomos são elevados na presença de fatores estressantes (NOGUEIRA et al., 2008 apud LUZ et al., 2014).

De acordo com Andrade et al., (2016), o vitiligo pode influenciar no estado emocional do paciente, para isso torna-se de suma importância que o profissional de enfermagem compreenda de forma significativa, como essa doença age, e como pode influenciar no convívio do portador, para que assim possa suprir as necessidades existentes relacionas tanto a manifestação clínica da doença, como os fatores que possam ser associados de forma negativa e positiva.

A enfermagem possui um papel voltado para os cuidados essências de todas as doenças, mas especialmente aquelas que possuem influencia psicológica no paciente, isso porque os profissionais dessa área possuem uma aproximação mais relevante com os pacientes, tanto na atenção primaria como no acompanhamento de tratamentos e em recuperações. (MACEDO et al, 2010).

\section{CONCLUSÃO}

O vitiligo não leva à incapacidade funcional, mas causa grande impacto psicossocial. O estresse relatado por diversos trabalhos é um dos fatores mais relevante no desenvolvimento da doença vitiligo.

Sobre o uso de tecnologias, destaca-se que, historicamente, o modelo assistencial para a saúde teve seu foco nas tecnologias, para as quais o saber médico estrutura o trabalho de outros profissionais. $\mathrm{O}$ atendimento decorrente dessa estrutura é centrado no ato prescritivo, hegemônico e produtor de procedimentos, garantindo aos pacientes a integralidade da atenção.

\section{REFERÊNCIAS}

1. ANDRADE, J. A; et al. Vitiligo: Avaliação histológica e clinica após curetagem sequencial. Revista $A B D$, v 82, n4, p. 12, julho de 2010. 
2. CASANOVA, N.; SEQUEIRA, S.; SILVA; V. M. Instituto Superior Texeira Gomes. Portugal.2009.

3. COSTA, M.C.C; et al. Perfil Comportamental de Crianças com Dermatoses Crônicas de Acordo com a Avaliação dos Cuidadores. Psico, v. 44, n. 2, pp. 167173, abr./jun. 2009.

4. DIAS, M.B. Vitiligocomo sintoma: A dificuldade no contato com as emoções. Revista de psicanálise, número especial, maio de 2005.

5. DIAS, N. G; et al. Fatores desencadeantes em dermatoses crônicas de crianças com perfis internalizantes e externalizantes segundo relato materno. Psico. Porto Alegre, 2016. V.47, n.1, p.46-55.

6. LUZ, L. L.; SANTOS, S. L.; PARTATA, A. K. Vitiligo e seu tratamento. Revista cientifica do ITPAc: Araguaína, v7, n3, pub.5, julho 2014.

7. MACEDO, A. C. B.; et al. Efeitos da aplicação do L.A.S.E.R. HeNe e do ultravioleta B no vitiligo. Fisioterapico. Mov., Curitiba, v. 25, n. 3, p. 481-488, jul. /Set. 2010.

8. MENEZES, M.; et al. Psicoterapia de criança com alopecia areata universal: desenvolvendo a resiliência. Paidéia, Vol. 20, N. 46, p. 261-267. Maio-ago. 2010.

9. NETO, A. T. M.; et al. Vitiligo: O problema que não está apenas na pele. Revista interdisciplinar do pensamento cientifico. Rio de Janeiro: Quiçamã; v 1, n 2, artigo $\mathrm{n}^{\circ}$ 18, julho/dezembro de 2015. p. 250-288.

10. NOGUEIRA, L. S. C.; ZANCANARO, P. C. Q.; AZAMBUJA, R. D. Vitiligo e emoções. Anais Brasileiro de Dermatologia. 2004.

11. SILVA, D. G.; MOREIRA, J. A.; PINTO, N. M. M. Vitiligo: Influencia na autoestima das pessoas afetadas. Revista de Enfermagem Integrada. Itapetinga. Unileste - MG, v2. Nov/Dez, 2009.

12. SILVA, D. R.Psicologia e saúde: Estresse percebido e qualidade de vida em dermatoses crônicas no contexto de saúde. In: Salão UFRGS 2015: SIC- XXVII Salão de iniciação Cientifica da UFRGS. Porto Alegre- RS, 2015. 